\title{
MAGNETIC CONFINEMENT OF RADIOTHERAPY BEAM-DOSE PROFILES
}

\author{
F.D. Becchetti, D.W. Litzenberg, J.M. Moran, T.W. O’Donnell, D.A. Roberts, B.A. Fraass, D.L. \\ McShan, and A.F. Bielajew, University of Michigan, Ann Arbor, MI 48109, USA
}

\section{Abstract}

We have used electron and photon beams from the 50 MV electron microtron at UM Hospital together with a large-bore 3.5T superconducting solenoid to demonstrate the magnetic confinement of $\mathrm{HE}$ electron and photon beam-dose profiles for typical radiotherapy beams. The HE electron beams in particular exhibit a large reduction in penumbra when entering a tissueequivalent phantom and, in addition, confinement of the secondary electrons produced by the primary beam. Likewise photon beams show a similar confinement of the dose from secondary electrons. While the results resemble features predicted from Monte Carlo calculations, there are a number of anomalous details in the actual experimental data which serve to illustrate the problems associated with practical clinical implementations. However the data suggest that in certain cases $\mathrm{HE}$ electrons may provide a cost-effective alternate to proton or HI radiotherapy beams and, also, improve the dose profile for HE photon beams.

\section{INTRODUCTION}

Monte Carlo simulations [1-5] and more recently, experiments [6] have shown that application of a strong magnetic field can provide a substantial improvement and control of the dose profile of clinical electron and photon radiotherapy beams. Other calculations also indicate some improvement in the dose profile for proton-therapy beams but to a much lesser extent than predicted for electron beams. The effect with proton beams, as well as photon beams, is in the confinement of the secondary electrons and recoil ions and not confinement or focusing of the primary beam itself. This would also presumably apply to neutron-beam therapy, or boron-neutron capture therapy (BNCT) where the secondary particles would also be confined.

As noted below, tissue-air interfaces (e.g. lung) can cause problems in conventional radiotherapy due to beam spreading at the air-tissue interface regions. In particular, however, we wish to show that the magnetic confinement and focusing of primary high-energy electron beams could make these beams more useful in radiotherapy and, perhaps, provide a viable alternative to proton- or heavy-ion beam therapy in some treatments. A number of technical challenges remain, but much of the technology developed for construction of compact superconducting cyclotron magnets, including gantry-mounted cyclotrons [7] can likely be utilized to develop clinical devices.

\section{HIGH-ENERGY ELECTRON-BEAM FOCUSING AND DOSE CONFINEMENT}

In order to examine some of the technical issues involving the utilization of magnetic focusing and dose confinement in a clinical setting a series of experiments were performed at the Radiation Oncology Department at the University of Michigan Hospital. The Scantronix MM50 $50 \mathrm{MV}$ electron racetrack microtron coupled to an isocentric G50 treatment gantry which includes a photon converter and collimator head was used to produce HE electron and photon beams [8].

A large warm-bore $(20 \mathrm{~cm})$ by $40 \mathrm{~cm}$ long air-core super-conducting solenoid [9] was installed $2.5 \mathrm{~m}$ from the gantry vacuum window with the primary electron beams passing through a helium gas-filled bag to reduce beam scatter (Fig. 1). Beam profiles without a phantom and dose profiles in a polystyrene phantom were recorded on x-ray films suitably located in the phantom. These were then scanned and digitized. Complete details can be found in Ref [6].

The dose profiles are deduced from the digitized-film optical-density information. Such data are shown in Figs. 2 and 3 for collimated $50 \mathrm{MV}$ and $20 \mathrm{MV}$ electron beams in the phantom. Dose profiles for the $20 \mathrm{MeV}$ electron beam $(\mathrm{B}=0$ and 3T) passing through a $5 \mathrm{~cm}$ diameter collimator located at the entrance of the phantom are shown in Fig 3. The data shown in Fig. 3 include the film-density to dose conversion but otherwise show the same features observed with the 50 $\mathrm{MeV}$ beam (Fig. 2). Optical-density profiles (closely related to dose) taken along the central beam axis and perpendicular to the beam axis at selected points for the $50 \mathrm{MeV}$ beam are shown in Fig. 4

As expected, the $3 \mathrm{~T}$ field results in a substantial focusing of the primary electron beam and, in addition confines the resulting dose profile. In particular the large penumbra typically associated with electron beams, which limits their use in radiation therapy, is significantly reduced. Likewise one expects the tissueair interface problem to also be minimized [10], especially if Tesla-level fields can be applied. 
As noted in [6] the experimental data show several features different than predicted by Monte Carlo (MC) calculations due to differences in the assumed field profiles and, also, due to some important features such as primary-beam focusing not included precisely in the MC calculations. In particular the Bragg-like dose profile predicted [3] is not seen to the extent predicted. Nonetheless, the dose profiles for the confined highenergy electron beams exhibit features similar to those for proton- therapy beams in tissue especially when the latter are modulated to treat $\mathrm{cm}$-size tumors. This suggests that focused and confined electron beams could provide a relatively low-cost alternative to proton therapy for certain treatments.

\section{TECHNICAL ISSUES}

Obviously there are a number of technical issues which must be addressed for the clinical application of magnetic confinement of radiotherapy beams. In our experiments the steel yoke of the gantry (Fig. 1) caused a perturbation of the solenoid field [6] so obviously a non-magnetic gantry would be preferable. In addition, the magnetic field affected the beam optics in the gantry resulting in a non-uniform treatment beam. A large-bore magnet would be needed for patient treatment depending on the area being treated and the treatment plan. Large-bore $\mathrm{NbTi}$ (up to 9T) and, recently, $\mathrm{NbSn}$ (up to $14 \mathrm{~T}$ ) are available although $\mathrm{NbSn}$ magnets remain expensive.

Alternately, large split-coil magnets perhaps with cryocoolers mounted together with the beam delivery system on a suitable gantry also appear feasible. It should be noted that the axial fringe field of the magnet actually adds focusing for an on-axis beam, e.g. a beam from a gantry-mounted linac if it's on the magnet axis.

\section{FUTURE WORK}

One issue that we hope to study is the effect of magnetic confinement on the radiobiological effect (RBE) of various radiotherapy beams. This is in addition to the dose confinement as the latter as presently measured (x-ray film) doesn't include any RBE enhancement e.g. from the confinement of secondary delta rays.

Likewise, in conjunction with imaging studies using positron-emission tomography (PET), it has been shown [11] that a magnetic field can be used to confine the $\beta^{+}$emission from the PET isotope being imaged and hence improve the PET spatial resolution. This might also have applications in the PET imaging of $\beta^{+}$ emitters produced in proton-beam[12] or heavy-ion beam radiotherapy. Related to this is the possibility of using magnetic confinement in selected brachytherapy treatments where, again, one might be dealing with high-energy hence long-range charged, radioactivedecay particles $\left(\beta^{-}, \beta^{+}\right.$, or $\alpha^{\prime}$ s).

\section{ACKNOWLEDGEMENTS}

The authors thank the staff at UM Hospital Radiation Oncology for their assistance. This work was supported in part by NSF Grant PHY9804878.

\section{REFERENCES}

[1] C.C. Shih, "High Energy Electron Radiotherapy in a Magnetic Field," Med. Phys. 2 (1975) 9-13.

[2] D.P. Whitmire, D.L. Bernard and M.D. Peterson, "Magnetic Modification of the Electron-dose Distribution in Tissue and Lung Phantoms," Med. Phys. 5 (1978) 409-17.

[3] M.S. Weinhous, R. Nath and R.J. Schultz, "Enhancement of Electron Beam Dose Distributions by Longitudinal Magnetic Fields: Monte Carlo-Simulations and Magnet System Optimization," Med. Phys. 15 (1985) 598-603.

[4] A.F. Bieljajew, "The Effect of Strong Longitudinal Magnetic Fields on Dose Deposition From Electron and Photon Beams," Med. Phys. 20 (1993) 1171-9.

[5] X.A. Li, L. Reiffel, L. Chu and S. Naqvi, "Conformal Photon-beam Therapy with Transverse Magnetic Fields: A Monte Carlo Study," Med. Phys. 28 (2001) 127-33.

[6] D.W. Litzenberg, A.F. Benedick, D.L. McShan, T.W. O'Donnell, D.A. Roberts, F.D. Becchetti, A.F. Bielajew and J.M. Moran, "An Apparatus for Applying Strong Longitudinal Magnetic Fields to Clinical Photon and Electron Beams," Phys. Med. Biol. 46 (2001) N105-N115.

[7] H. Blosser, et al., "Superconducting Cyclotron for Medical Application," IEEE Transactions on Magnetics, Vol. 25, No. 2 (March 1989)17461754.

[8] M. Karlsson, H. Nyström, and H. Svensson, "Electron Beam Characteristics of the $50-\mathrm{MeV}$ Racetrack Microtron," Med. Phys. 19 (1992) 30715.

[9] R.L. Stern, et al., "Tests of a Large Air-core Superconducting Solenoid as a Nuclear-reactionproduct Spectrometer," Rev. Sci. Instrum. 58 (1975) 1682-93.

[10] S.A. Nagyi, X.A. Li, S.W. Ramahi, J.C. Chu and S.J. Ye, "Reducing Loss in Lateral Chargedparticle Equilibrium Due to Air Cavities Present in X-ray Irridiated Media by Using Longitudinal Magnetic Fields," Med. Phys. 28, (2001) 603-611.

[11] R. Raylman, "Reduction of Positron Range Effects by the Application of a Magnetic Field for use with Positron Emission Tomography," Ph.D. Thesis (University of Michigan, 1991).

[12] D.W. Litzenberg, D.A. Roberts, M.Y. Lee, K. Pham, A.M. VanderMolen, R. Ronningen, and F.D. Becchetti, "On-line Monitoring of Radiotherapy Beams: Experimental Results with Proton Beams," Med. Phys. 26, Issue 6 (1999) 992-1006. 


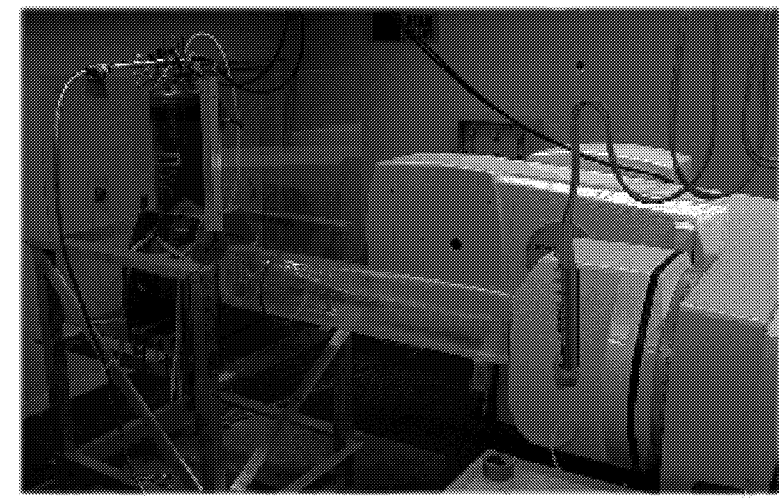

Figure 1: Experimental arrangement showing the superconducting solenoid with phantom (left) and helium bag at exit G50 microtron radiotherapy gantry (right).

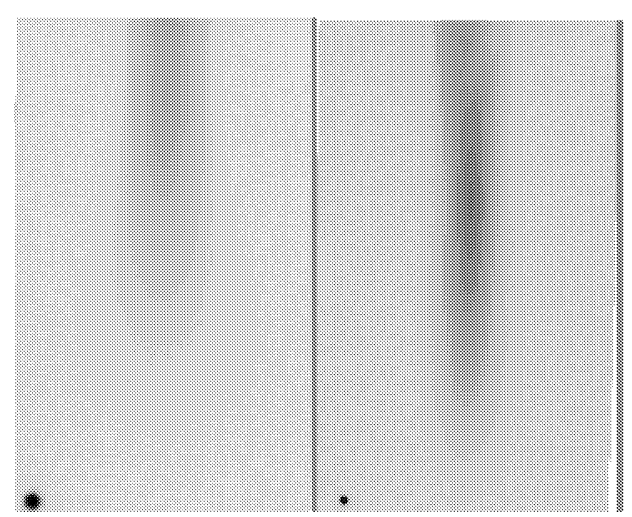

Figure 2: Film-density scans of a $5 \mathrm{~cm}$ diameter collimated $50 \mathrm{MeV}$ electron beam in a polystyrene phantom without (left) and with (right) a 3T applied field.

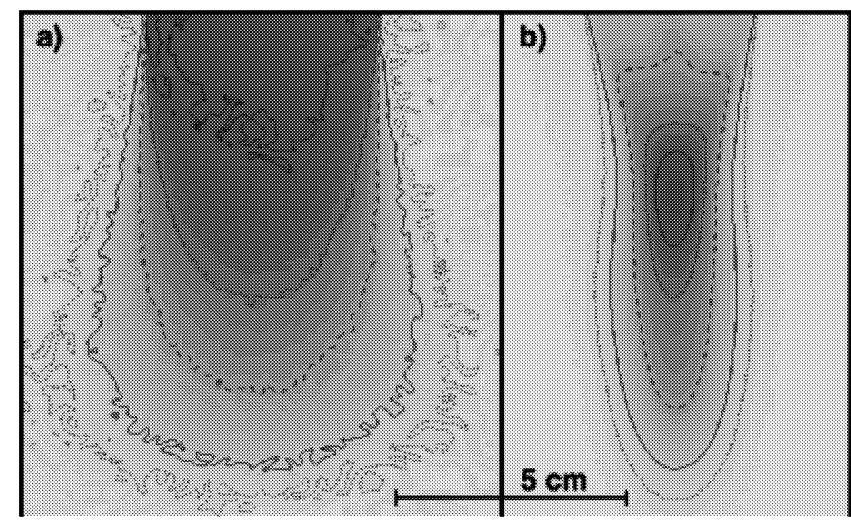

Figure 3: Isodose profiles for a $5 \mathrm{~cm}$ diameter $20 \mathrm{MV}$ electron beam in a polystyrene phantom without (left) and with (right) an applied 3T field along the incident beam direction (vertical axis).
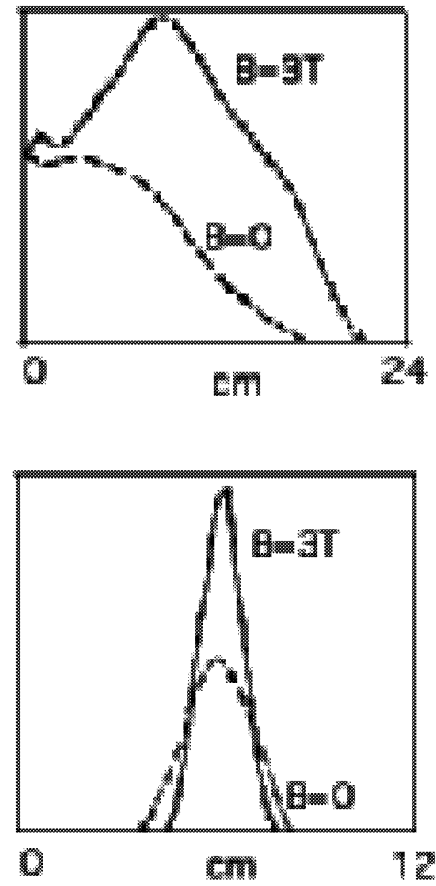

Figure 4: Optical density scans, proportional to dose, taken along the beam axis (top) and perpendicular to the beam axis (bottom) near the maximum in the magnetically-confined $50 \mathrm{MeV}$ electron beam profile (Fig. 2) 